\title{
A Network Pharmacology Approach to Investigate the Underlying Mechanisms of Alpinia Katsumadai Hayata on Acne Vulgaris
}

\author{
Li Wang ${ }^{1 *}$, Yang $\mathrm{Nie}^{2}$, Huifang Chen (Co-first Author) ${ }^{1^{*}}$, Jun $\mathrm{Sun}^{3}$, Mingyue $\mathrm{Hu}^{1}$, Youyi Xu (Corresponding Author) ${ }^{1}$ and \\ Shaozhen Chen (Corresponding Author) ${ }^{1}$ \\ ${ }^{1}$ College of Pharmacy, Guangdong Lingnan Institute of Technology, Guangzhou 510663, China; \\ ${ }^{2}$ School of Pharmaceutical Engineering, Guangdong Food and Drug Vocational College, Guangzhou 510520, China; \\ ${ }^{3}$ Guangzhou Institute of Advanced Technology, Chinese Academy of Sciences, Guangzhou 511458, China \\ *The Co-first Author: Huifang Chen - College of Pharmacy, Guangdong Lingnan Institute of Technology, Guangzhou 510663, China; \\ Email: chenhf1968@163.com
}

Author Contributions * Li Wang and Huifang Chen contribute equally.

\begin{abstract}
The Alpinia katsumadai Hayata (Doukou, DK) is a traditional Chinese medicine that has shown superior anti-inflammatory property, which is widely used in the food and commodity industry. A network pharmacology analysis was performed to identify the potential anti-acne compounds, hub therapeutic targets, and the key pathways via TCMSP, BATMAN, CTD, PDB and PubChem databases. Finally, the "compoundtarget-pathway" network was constructed. The study found total 7 active compounds, including quercetin, (2R)-5,7-dihydroxy-2-phenylchroman-4-one, dehydrodiisoeugenol, (2R)-7-hydroxy-5-methoxy-2phenylchroman-4-one, Pinocembrin, and 1,7-diphenyl-5-hydroxy-6-hepten-3-one alpinolide peroxide. In addition, 30 therapeutic targets, and 4 hub therapeutic targets of the DK were identified. The biological processes were primarily related to inflammatory response, response to oxidative stress, regulation of insulin secretion, etc. Which was significantly associated with ten pathways including the PI3K-Akt signaling pathways, VEGF signaling pathways, etc. Furtherly, the 4 hub targets AKT1, F2, AR, and PTGS2 with higher connectivity in PPI network were verificated though molecular docking, which once again proved that these targets are potential targets of their corresponding chemical molecules. Therefore, DK might have a synergistic effect on the anti-inflammatory effects via the various active compositions, targets and signaling pathways.
\end{abstract}

\section{Introduction}

Acne vulgaris is an inflammatory disease of hair follicle sebaceous glands, which is chronic and self limited. This is a very common skin disease, mainly in the face of inflammatory and non inflammatory lesions, but may also occur in the upper arm, trunk and back. At present, the highest incidence rate of skin disease is popularly known as acne ${ }^{[1]}$. Traditional Chinese medicine is also known as "feifeng acne", "acne rash", "facial blister" and so on. In the third chapter of Su Wen . Qi Qi Tong Tian Lun, it is said that "perspiration and dampness bring acne rash." Sweat is the wind, cold is the wind, and depression is the acne ${ }^{[2]}$ "Elbow reserve urgent prescription" said: "young gas full, blisters on the face", put forward that young people because of the blood is just, Qi and blood full, is born of this disease. $\mathrm{Xu}$ Kechang, Qing Dynasty, wrote in the complete book of surgical treatment and syndrome: "feifeng acne, broken red pimples on the face and nose, shaped like millet crumbs, breaking out white powder juice, it is appropriate to use sulfur cream, apply it after washing the face, and it will heal in a few days." Throughout the views of ancient doctors, treatment is mostly based on the lung meridian. In the understanding of etiology and pathogenesis, the view of Huangdi Neijing Suwen occupies an absolutely dominant position ${ }^{[3]}$. Network pharmacology is a new discipline based on the theory of systems biology, which can select specific signal nodes for multi-target drug molecular design by network analysis of biological system ${ }^{[4]}$.Chen Xiangjun et al. Screened 20 active components from Angelica dahurica through network pharmacology analysis, acting on 55 targets, mainly through regulating PI3K / Akt, nuclear factor kappa B $(\mathrm{NF}-\kappa \mathrm{b})$, mitogen activated protein kinase (MAPK), fibroblast growth factor receptor 2 (FGFR2) and other signal pathways to play the role in the treatment of acne [5]. Network pharmacology analysis showed that the active components of Salvia miltiorrhiza could interfere with acne vulgaris biological network by acting on 5LOX, lta4h, h1r, HDAC4, $5 \alpha-\mathrm{R} 1$, steroid sulfatase and 
CYP19A1. Among them, tanshinone I, salvianolic acid B and tanshinone IIA may be the main anti androgen and antiinflammatory components. Therefore, Salvia miltiorrhiza may have certain anti androgen and anti-inflammatory pharmacological effects in the treatment of acne vulgaris ${ }^{[6]}$. This study explored the mechanism of cardamom in the treatment of acne vulgaris through the methods of network pharmacology and molecular docking, so as to deepen our understanding of the theoretical basis of cardamom in the treatment of acne vulgaris.

\section{Materials and methods}

\subsection{Screening of active components from cardamom}

Using tcmsp database

（ http://lsp.nwu.edu.cn/browse.php?qc=herbs ) Obtain the ingredient information of the drug Doukou, including the number of ingredients and the molecular name of the ingredient Molecular weight (MW), lipid water partition coefficient (alogp), number of hydrogen bond donor receptor (hdon / HACC), oral bioavailability (OB), intestinal epithelial permeability (Caco-2), blood-brain barrier (BBB), drug similarity (DL), drug half-life (HL), etc. ADME parameters were further used to screen out possible small drug molecules. ADME data were obtained from tcmsp database. "ADME", or "pharmacokinetics", is to study the absorption, distribution, metabolism and excretion of exogenous chemicals. ADME parameters include oral bioavailability (OB), drug similarity (DL) and half-life (HL). In this paper, the threshold values of ob $\geq$ $30 \%$ and $\mathrm{DL} \geq 0.18$ were set for drug component screening.

\subsection{Prediction of target proteins corresponding to active components of cardamom}

Using Batman database ( http://bionet.ncpsb.org/batman$\mathrm{tcm} /$ ) $^{[8]}$ To predict the target proteins of small chemical molecules, Batman uses the similarity based method to predict the potential targets of traditional Chinese medicine ingredients. Its core idea is to rank the potential targets according to the similarity between the interaction of potential targets and known targets. Firstly, the targets in the databases of drugbank, KEGG and TTD will be given, and then the predicted targets will be ranked in descending order. Here, we select the target eggs with each molecule score greater than 20 White, that is, the threshold is set to score cutoff $=20$. For the components that do not exist in Batman, tcmsp database is directly used to predict the target of corresponding drug components.

\subsection{Screening disease-related targets for target protein cross validation}

Mount Desert Island biological laboratory has published CTD 2018 update (comparative toxicology) Database, http://ctdbase.org/ ) [9] To promote understanding of how environmental risks affect human health, it provides information on chemical gene / protein interactions, chemical disease and gene disease relationships to help develop disease mechanisms about environmental impacts.

In the CTD database, "acne vulgaris" was used as the key word to search for acne vulgaris related genes, and according to the order of the inference score, the intersection of those with a score of more than 10 and the target protein predicted in the previous step was selected to narrow the range of target protein Score is calculated by the logarithmic transformation product of two common neighborhood statistical data to evaluate the functional correlation between proteins in protein interaction network.

\subsection{Construction of cross protein PPI network}

Using online tool string ( https://string-db.org/ ) ${ }^{[10]}$ The PPI relationship of these cross target proteins was analyzed, and the species was Homo sapiens. The parameter PPI score is set to 0.9 (highest confidence), which requires that all protein nodes involved in the interaction should be included in the above differential genes. After getting PPI relation pairs, we use Cytoscape software (version 3.4.0, http://chianti.ucsd.edu/cytoscape-3.4.0/ ) Construct the network diagram.

Using the cytonca plug-in (version 2.1.6, http://apps.cytoscape.org/apps/cytonca ) ${ }^{[11]}$ The node connectivity degree centrality (DC) is analyzed, and the parameter is set to without weight. According to the network topological properties of each node, the hub protein, which is an important node in PPI network, is obtained.

\subsection{PPI module analysis}

In the protein interaction network, the cluster analysis is used to identify the functional modules, and the network topology and the relationship between network components are obtained. Finally, the protein complexes or functional modules with biological significance are obtained. Therefore, we use the mcode plug-in of Cytoscape software, and the parameters are set to the default (degree cutoff $=2$, node score cutoff $=0.2$, $\mathrm{k}$-core $=2$, Max.Depth =The PPI network module mining is carried out. Similarly, go BP (go biological processes) and KEGG are carried out for each module using David tool Path enrichment analysis showed that the number of enriched genes $>=2$ and p.value $<0.05$ were significantly enriched term.

\subsection{Construction of network pharmacology network}

Based on the drug component target protein pathway, the pharmacological network was constructed by using Cytoscape. Objective to demonstrate the pathway regulation mechanism of related target proteins of important components in traditional Chinese medicine. 


\subsection{Molecular docking}

The hub protein obtained from PPI network analysis was used as the key target to find its corresponding chemical small molecule in the final network pharmacology, and it was used as the key small molecule.

Through PDB database ( http://www.rcsb.org/ ) Download the PDB format file of the complexes of key target proteins and other ligands for subsequent research.

PubChem database

( https://pubchem.ncbi.nlm.nih.gov/ ) ${ }^{[12]}$ It includes three sub databases: PubChem bioassay library is used to store biochemical experimental data, which mainly comes from high-throughput screening experiments and scientific and technological literature; PubChem compound library is used to store the chemical structure information of compounds; PubChem substance library is used to store the chemical structure information of compounds It is used to store the original data of compounds uploaded by institutions and individuals. In this study, the molecular structure of key small molecules was downloaded from PubChem compound database in SDF format and converted to PDB format by PyMOL (version 2.0 Schr > dinger, LLC.) for subsequent molecular docking.

The possibility of molecular docking between key small molecules and targets was studied by autodock software. Autodock is an open source molecular simulation software, which is mainly used to perform ligand protein docking. It is developed and maintained by Olson Laboratory of Scripps Research Institute, and the latest version is autodock 4.2. Autodock 4.2.6 was used for molecular docking.

\section{Results}

\subsection{Determination of important active components in cardamom}

According to the method part, we searched tcmsp database with "cardamom" as the key word, and got 71 kinds of chemical components of cardamom. According to the threshold screening described in the method section, 14 important chemical components were obtained, as shown in Table 1.

Table 1 The major active compounds of Alpinia Katsumadai Hayat

\begin{tabular}{|c|c|c|c|}
\hline Mol ID & Molecule Name & $\begin{array}{l}\text { Bioavail } \\
\text { ability } \\
\text { (OB) }\end{array}$ & Generic property (DL) \\
\hline \multirow{3}{*}{ MOL000235 } & 1,7-diphenyl-3,5-dihydroxy-1- & & 0.18 \\
\hline & heptene & 49.01 & \\
\hline & (3R,5S)-1,7-di(phenyl)heptane- & & 0.18 \\
\hline \multirow[t]{2}{*}{ MOL000240 } & 3,5-diol & 48.35 & \\
\hline & (2R)-5,7-dihydroxy-2- & & 0.18 \\
\hline \multirow[t]{2}{*}{ MOL000246 } & phenylchroman-4-one & 46.08 & \\
\hline & 1,7-diphenyl-5-hydroxy-6-hepten- & & 0.18 \\
\hline MOL000238 & 3-one & 32.65 & \\
\hline MOL000243 & alpinolide peroxide & 87.67 & 0.19 \\
\hline \multirow[t]{2}{*}{ MOL000230 } & Pinocembrin & 57.56 & 0.20 \\
\hline & $\begin{array}{l}\text { (2R)-7-hydroxy-5-methoxy-2- } \\
\text { phenylchroman-4-one }\end{array}$ & 55.23 & 0.20 \\
\hline MOL000006 & luteolin & 36.16 & 0.25 \\
\hline MOL000242 & 7-O-Methyleriodictyol & 56.56 & 0.27 \\
\hline MOL000098 & quercetin & 46.43 & 0.28 \\
\hline MOL000258 & dehydrodiisoeugenol & 56.84 & 0.29 \\
\hline \multirow[t]{2}{*}{ MOL000239 } & Jaranol & 50.83 & 0.29 \\
\hline & $\begin{array}{c}(4 \mathrm{E}, 6 \mathrm{E})-1,7-\mathrm{bis}(3,4- \\
\text { dihydroxyphenyl)hepta-4,6-dien-3- }\end{array}$ & 33.06 & 0.31 \\
\hline \multirow[t]{2}{*}{ MOL000224 } & one & & \\
\hline & $\begin{array}{c}\text { 5-[(2R,3R)-7-methoxy-3-methyl- } \\
\text { 5-[(E)-prop-1-enyl]-2,3- } \\
\text { dihydrobenzofuran-2-yl]-1,3- } \\
\text { benzodioxole }\end{array}$ & 65.55 & 0.40 \\
\hline
\end{tabular}

\subsection{Cross validation of component target proteins and disease-related targets and construction of drug component target network}

According to the method part, the important chemical components screened from tcmsp database were predicted by Batman online tool and tcmsp database, and the targets with scores of more than 20 were selected for each component, among which 10 important components 
had target records, a total of 129 targets.

Based on the method part, we take the intersection of 129 targets related to acne vulgaris in CTD database and the influence score is greater than 10 , and finally get 41 intersection related targets, as shown in Figure 1 Wayne diagram, which is defined as the key target of cardamom in the treatment of acne vulgaris. Furthermore, we screened out the chemical components corresponding to the 41 key targets and constructed the component target network, as shown in Figure 2. A total of 73 component target relationship pairs were obtained, including 9 chemical components and 41 targets, indicating that cardamom can treat acne vulgaris through multi-component and multitarget.

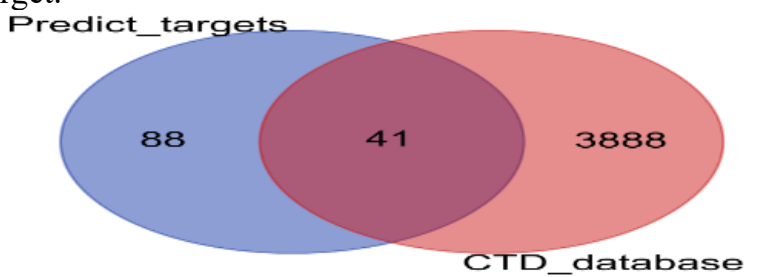

Fig.1 The venn diagram of cross-validated target proteins between targets of 10 candidate active components in DK and targets associated with Acne vulgaris.

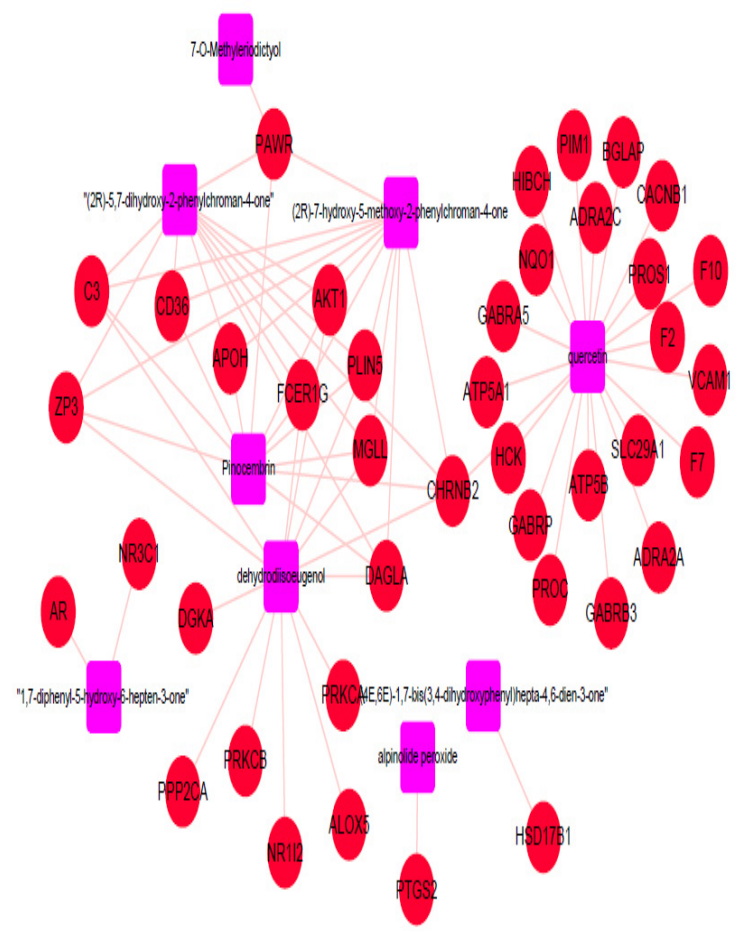

Fig.2 The compound-target network for DK on Acne vulgaris. The pink nodes represent candidate active compounds and the red nodes represent potential protein targets. The edges represent the interactions between them.

\subsection{PPI network construction}

In order to further understand the interaction between target proteins, we constructed a protein-protein interaction network (PPI network). Results as shown in Figure 3, there were 38 protein interactions and 74 protein pairs. The higher the connectivity of PPI network, the higher the critical value of protein, which is a potential hub protein. The top three are AKT1 (degree = 13), F2 (degree = 11), AR and PTGS2 (where AR and PTGS2 have the same connectivity, degree $=9$ ).

In order to cluster protein complexes or functional modules with biological significance, we use mcode algorithm to identify three sub modules, and get their network topology and the relationship between network components, as shown in Figure 3 (different colors represent different modules). Through go BP functional analysis and KEGG pathway enrichment analysis, we can understand the biological significance of these potential important target proteins. According to the p.value ranking, the top five go BP results of the module are shown in Figure 4, and the KEGG path results are shown in Figure 5. It can be seen from the figure that the main functions of the previously screened hub protein AKT1 and PTGS2 aggregation module 3 are platelet activation (GO: $0030168 \sim$ platelet activation), positive regulation of vasoconstriction (GO: 0045907 positive regulation of vasoconstriction), secret of insulin regulation (GO:0050796 insulin regulation), secret of adrenaline negative regulation (GO: $0032811 \sim$ adrenaline negative regulation), and secret of epidermal growth factor through G-protein White couple receptor signaling pathway activated receptors (GO:0006954 inflammatory response) and oxidative stress GO: $0006979 \sim$ oxidative stress response). KEGG pathway analysis mainly focused on VEGF signaling pathway (hsa04370), sphingolipid signaling pathway (hsa0471), cardiac myocyte adrenergic signaling pathway (hsa04261), cGMP PKG signaling pathway (hsa04022), neuroactive receptor (hsa04080) interaction, hsa0451: PI3K Akt signaling pathway.

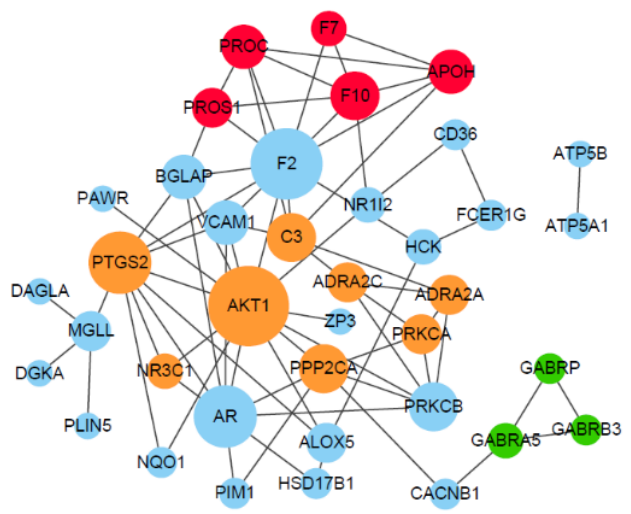

Fig.3 ,The Target protein PPI network of DK on Acne vulgaris. The dots are cross-validated target proteins. The edges represent the interactions between them and the dots size is proportional to their degree. The stronger the association, the larger the dots. Red dot represents module 1, green dot represents module 2 , and yellow dot represents Module 3 


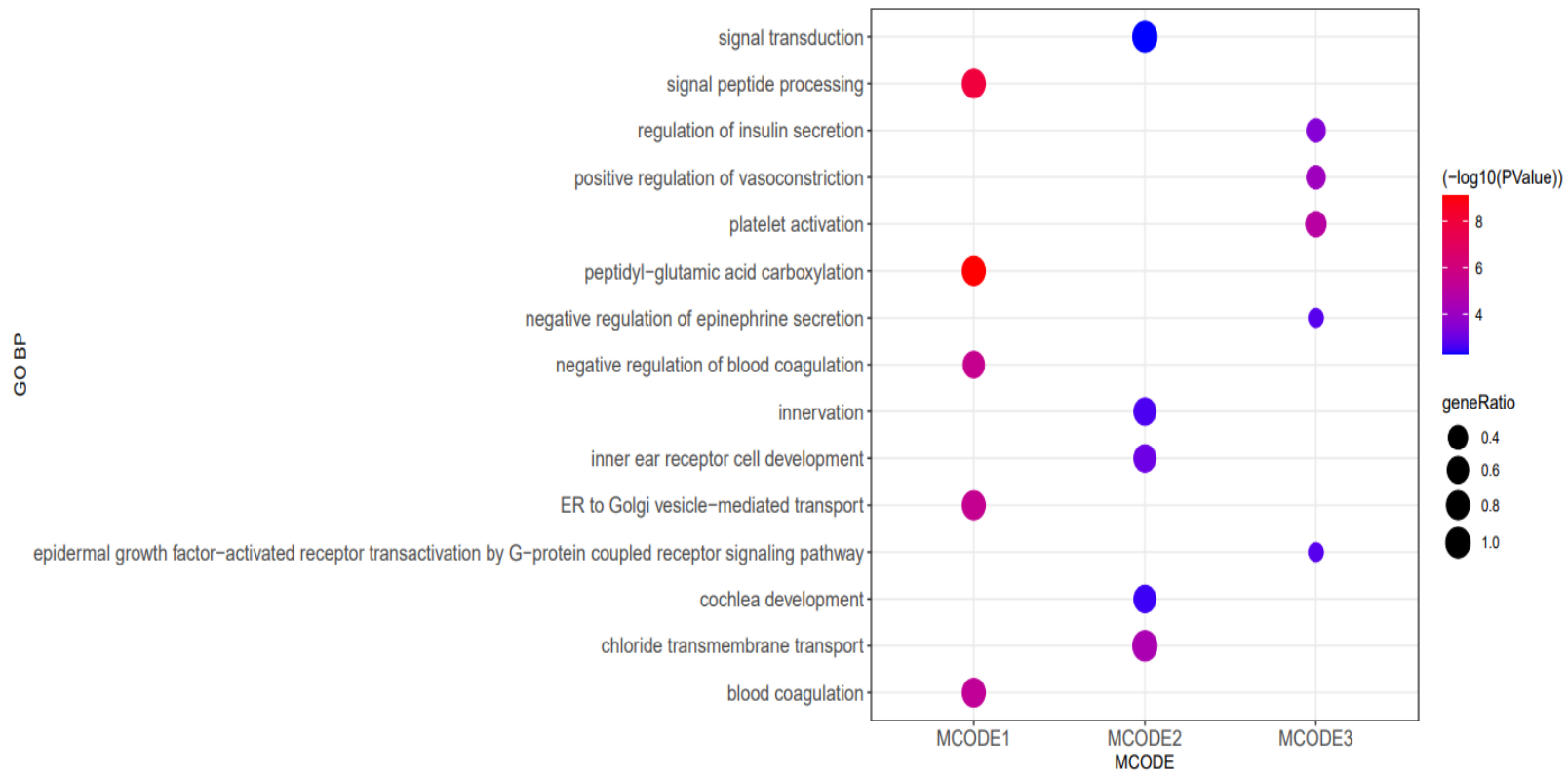

Fig.4, The 5 most significance of gene ontology enrichment analysis of therapy target genes in the three PPI network modules of DK on Acne vulgaris.

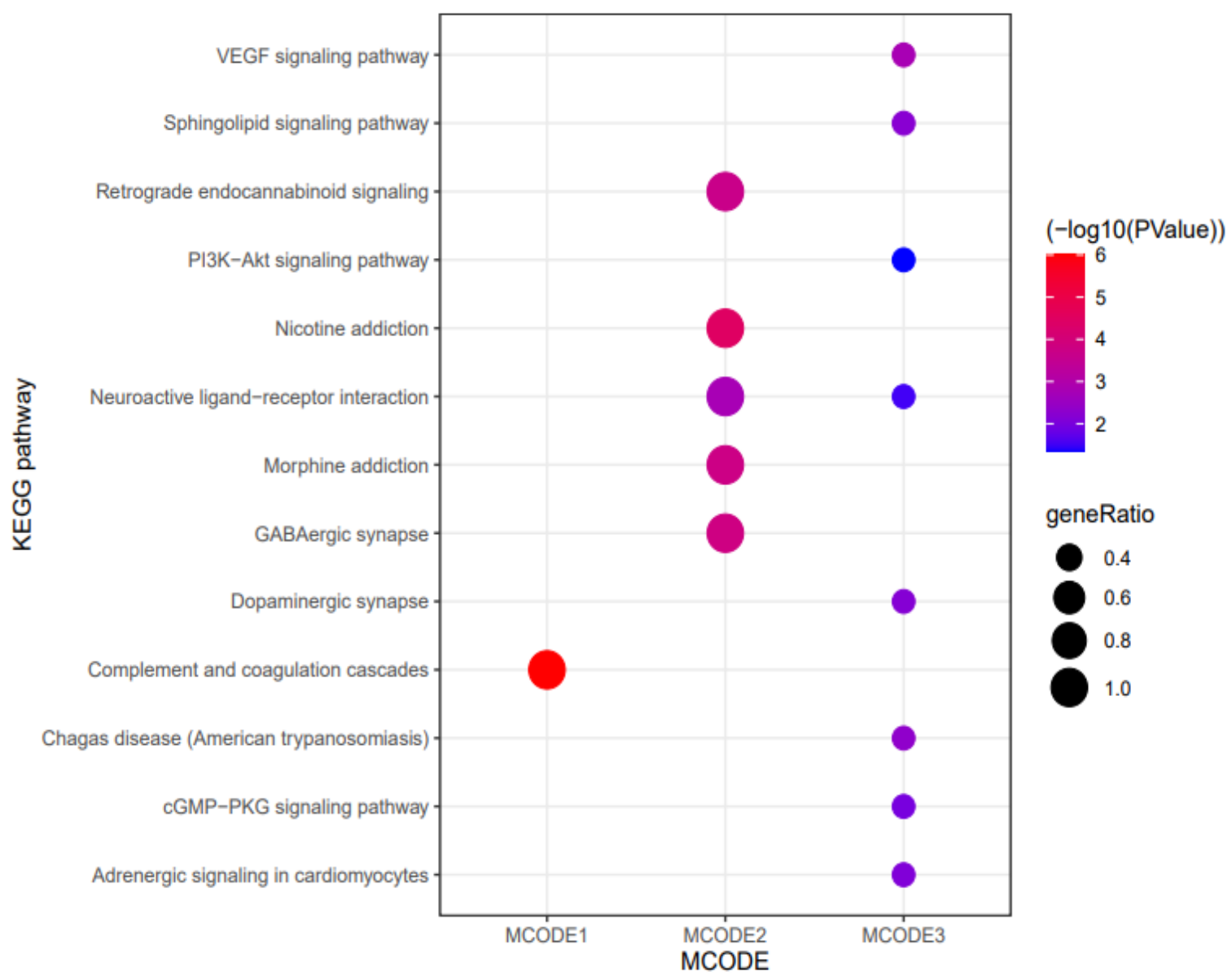

Fig.5 The 5 most significance of pathway enrichment analysis of therapy target genes in the three PPI network modules of DK on Acne vulgaris.

\subsection{Network construction of network pharmacology of cardamom}

The network pharmacology network was constructed based on "cardamom component target protein pathway", as shown in Figure 6. There are 58 nodes (including one cardamom node) and 172 relationship pairs in the network.
Among them, there are 7 chemical component nodes, 30 target protein nodes and 20 pathway nodes. The key targets (AKT1, F2, AR and PTGS2) are mainly involved in platelet activation, signal transduction, VEGF signaling pathway, sphingolipid signaling pathway, FC \& RI signaling pathway, neuroactive ligand receptor interaction, complement and coagulation cascade and 
serotonergic synapse.

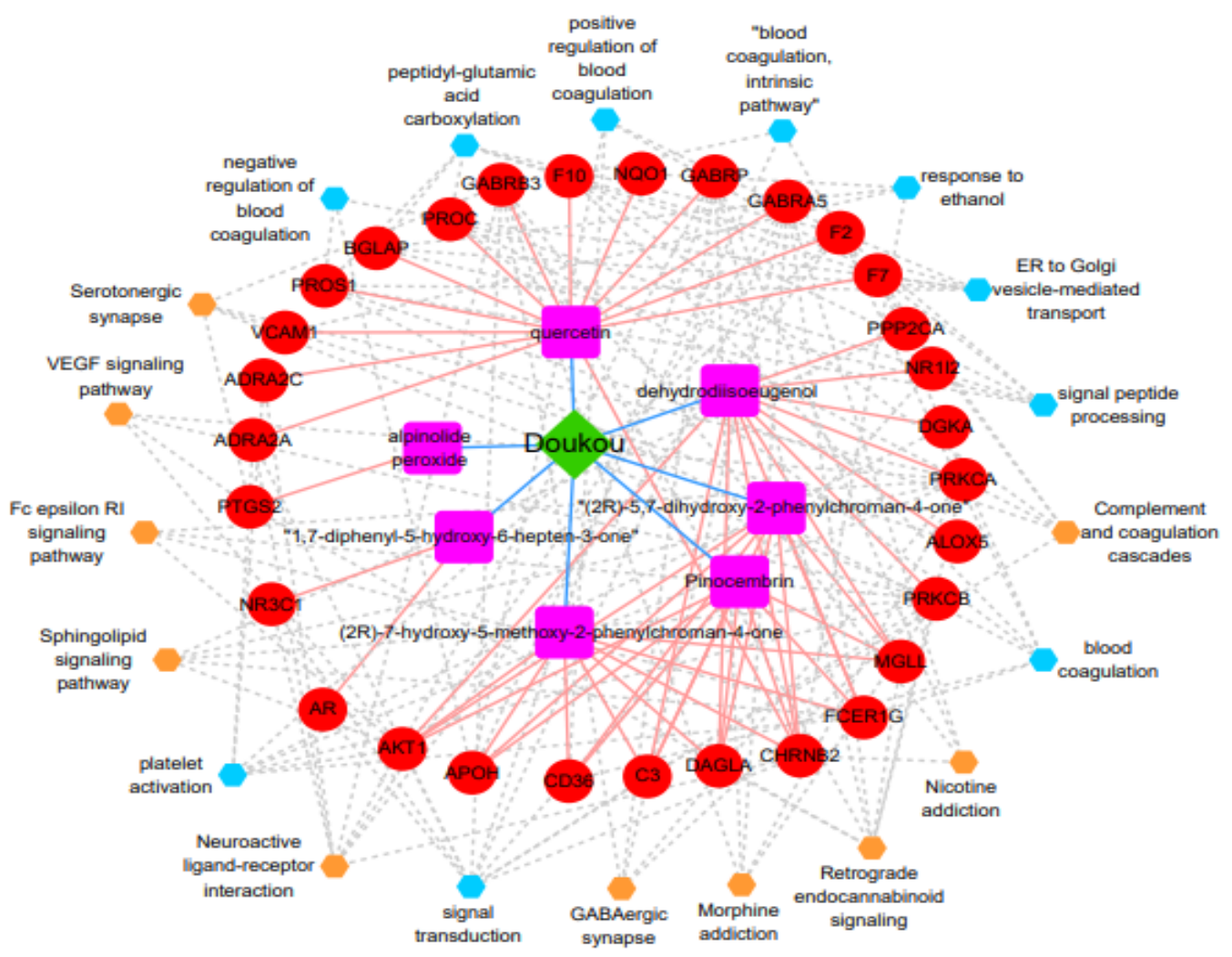

Fig.6 The compound -target-pathway network for DK on Acne vulgaris. The green nodes represent DK, the pink nodes represent compounds, the red nodes represent targets, the blue nodes represent GO BP terms, and the yellow nodes represent pathways. The blue edges represent the interactions between DK and compounds, the pink edges represent the interactions between targets and compounds, the gray edges represent the interactions between targets and GO functions or pathways.

\subsection{Verification results of molecular docking}

The experiment used the four most connected genes in PPI as the key targets: AKT1, F2, AR and PTGS2, and found their corresponding chemical molecules: dehydroisoeugenol (PubChem)_CID: 5379033), quercetin (PubChem)_CID: 5280343), 1,7-diphenyl-5-hydroxy-6hepten-3-one (PubChem)_ CID: 57520989), alpinolide peroxide (not included in PubChem, downloaded directly from tcmsp database). Among them, dehydroisobutanol, pinocembrin, (2R) - 7-hydroxy-5-methoxy-2phenylchroman-4-one and (2R) - 5,7-dihydroxy-2phenylchroman-4-one act on AKT1. The 3D structure of the four small chemical molecules is shown in Figure 7.

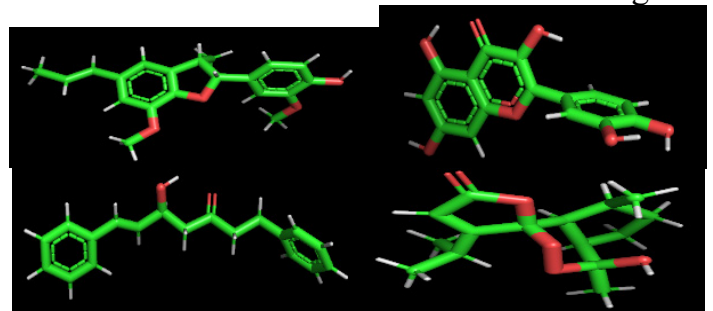

Fig.7 The The 3D structure of four key active compounds. Greens present carbon, grays present hydrogen, and red present oxygen. Left 1: dehydrodiisoeugenol,Left 12: quercetin, Left3:

1,7-diphenyl-5-hydroxy-6-hepten-3-one,Left4: alpinolide peroxide.

Secondly, the crystal structures of the complexes of four key target proteins with other ligands were downloaded from PDB database, from which water molecules were removed, and the 3D structure of protein was isolated. As shown in Figure 8.

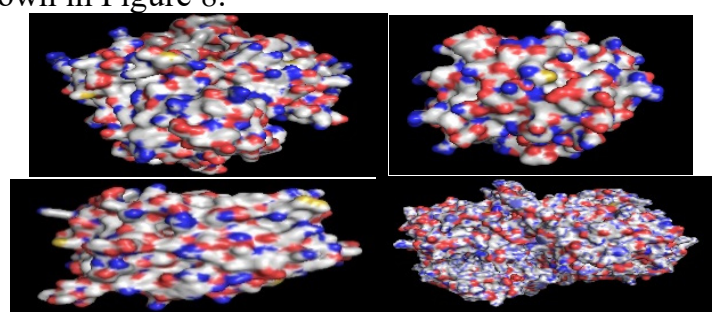

Fig.8 The structure-activity models based on 3D descriptors of four key active compounds. Upper left: AKT1, upper right: F2, lower left: AR, lower right: PTGS2.

According to the method part, the molecular docking of the above-mentioned small molecules and their corresponding target proteins is carried out through autodock 4.2.6. The smaller the binding energy is, the higher the possibility of binding is. Therefore, we select the model with the smallest binding energy as the best model and visually simulate the best model, as shown in Figure 9.

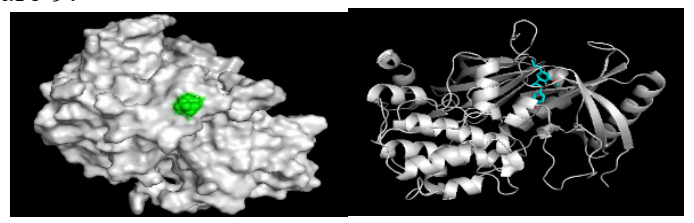




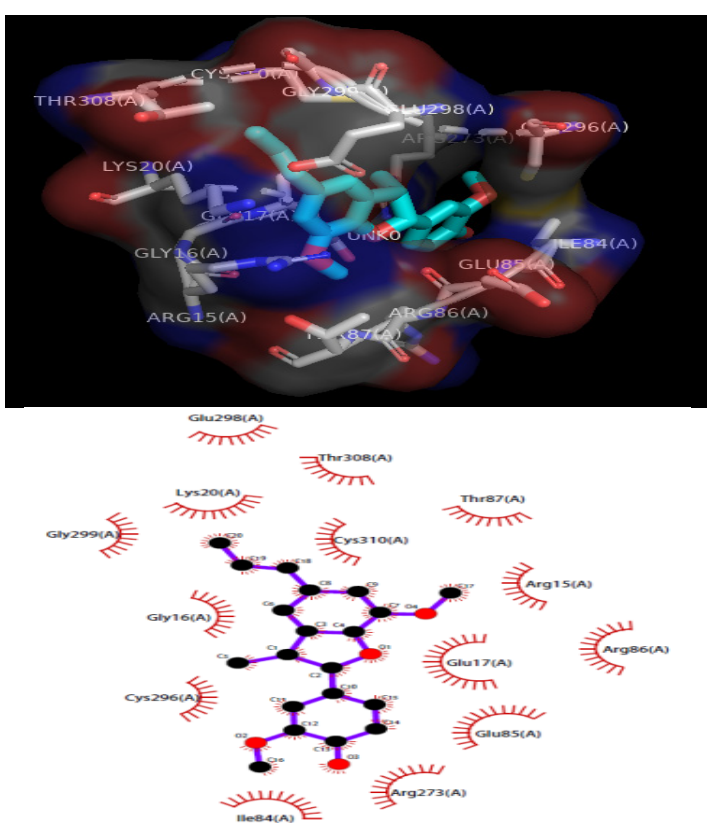

Fig.9-1 The Molecular models of DK binding to its predicted protein target AKT1.Left 1, left 2: a global profile of massive possible docking, The off-white color indicates the receptor

AKT1, the green sphere and the blue line both indicate the ligand small molecule dehydrodiisoeugenol. Left 3: the blue lines indicate the ligand small molecule dehydrodiisoeugenol, the text represents the amino acid residue that binds to AKTI.

Left 4: the black nodes represent ligand small molecule dehydrodiisoeugenol, the red semicircle represents the amino acid residue that binds to AKTI.
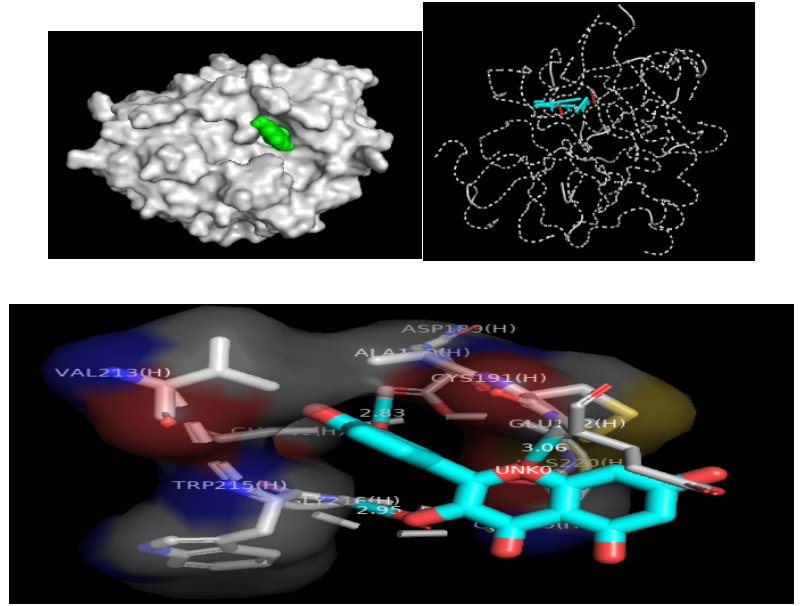

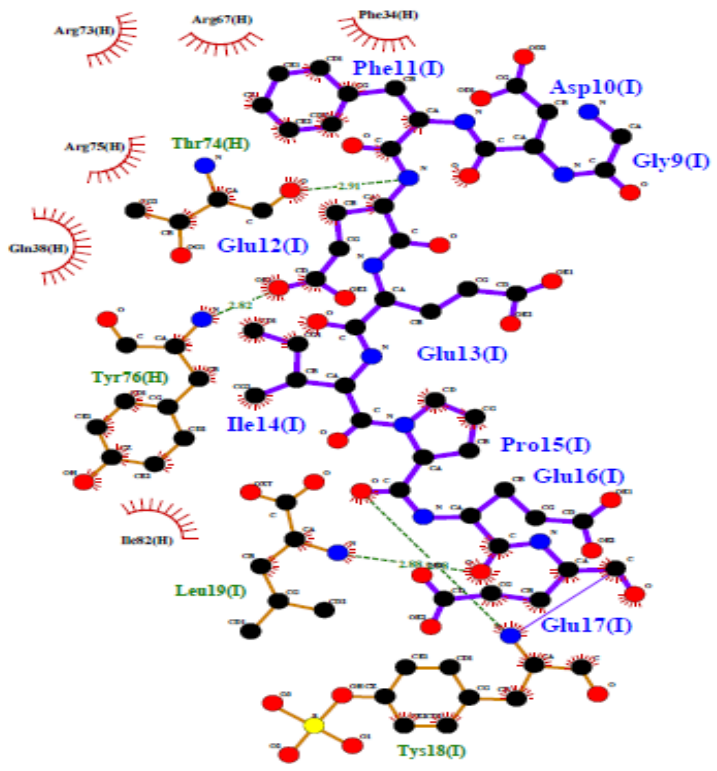

Fig.9-2 The Molecular models of DK binding to its predicted protein target F2. Left 1, left $2::$ a global profile of massive possible docking, The off-white color indicates the receptor F2, the green sphere and the blue line both indicate the ligand small molecule quercetin. Left 3: the blue lines indicate the ligand small molecule quercetin, the text represents the amino acid residue that binds to F2. Left 4: the black nodes represent ligand small molecule quercetin, the red semicircle represents the amino acid residue that binds to $\mathrm{F} 2$.

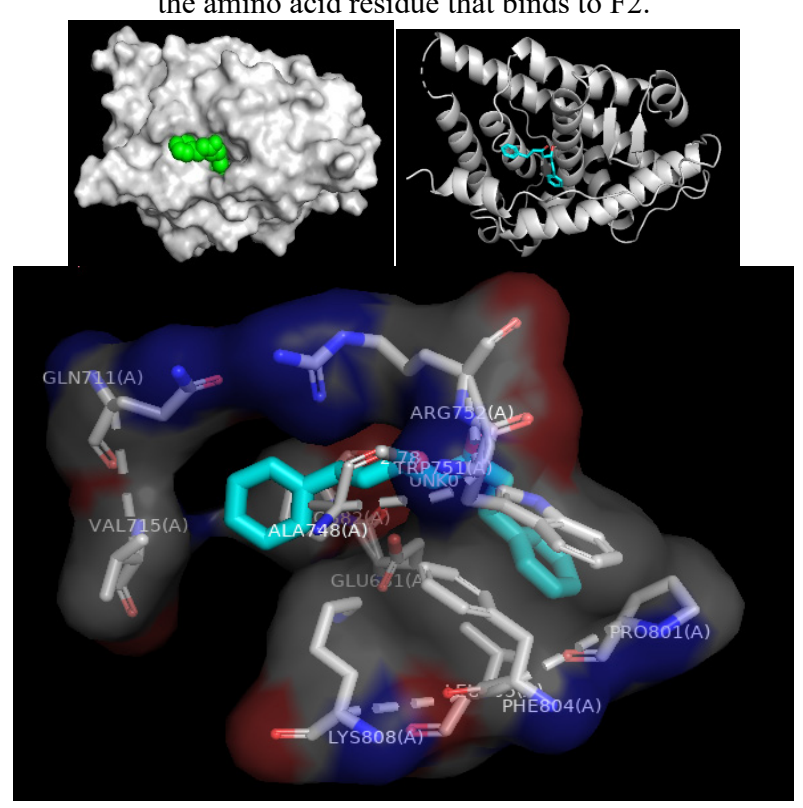




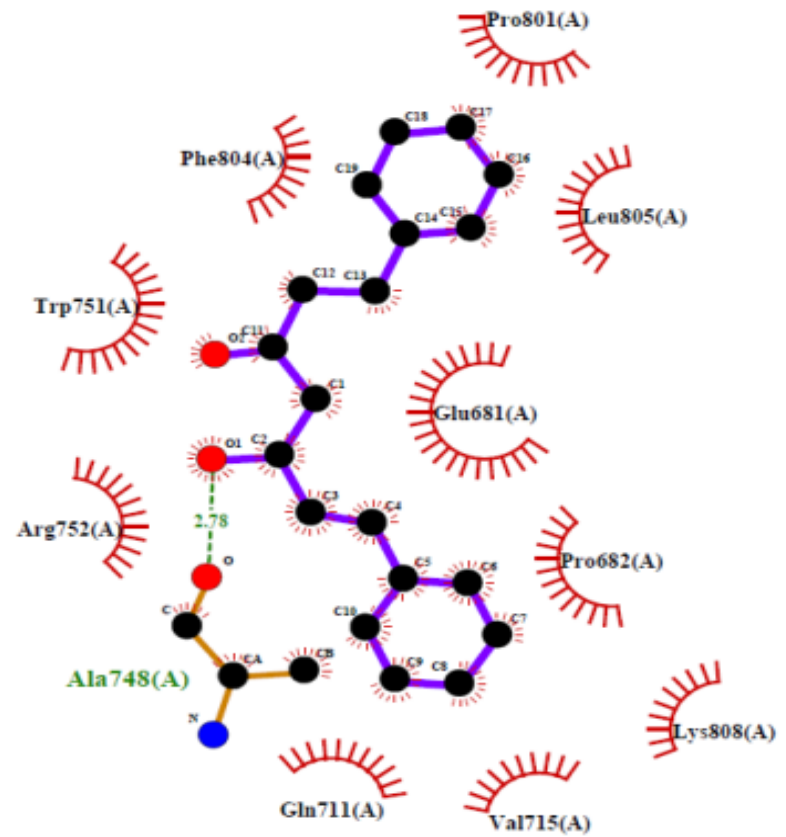

Fig.9-3 The Molecular models of DK binding to its predicted protein target AR. Left 1, left 2: a global profile of massive possible docking, The off-white color indicates the receptor AR, the green sphere and the blue line both indicate the ligand small molecule 1,7-diphenyl-5-hydroxy-6-hepten-3-one.Left3: the blue lines indicate the ligand small molecule 1,7-diphenyl-5-hydroxy-

6-hepten-3-one, the text represents the amino acid residue that binds to AR. Left4: the black nodes represent ligand small molecule 1,7-diphenyl-5-hydroxy-6-hepten-3-one, the red semicircle represents the amino acid residue that binds to AR.

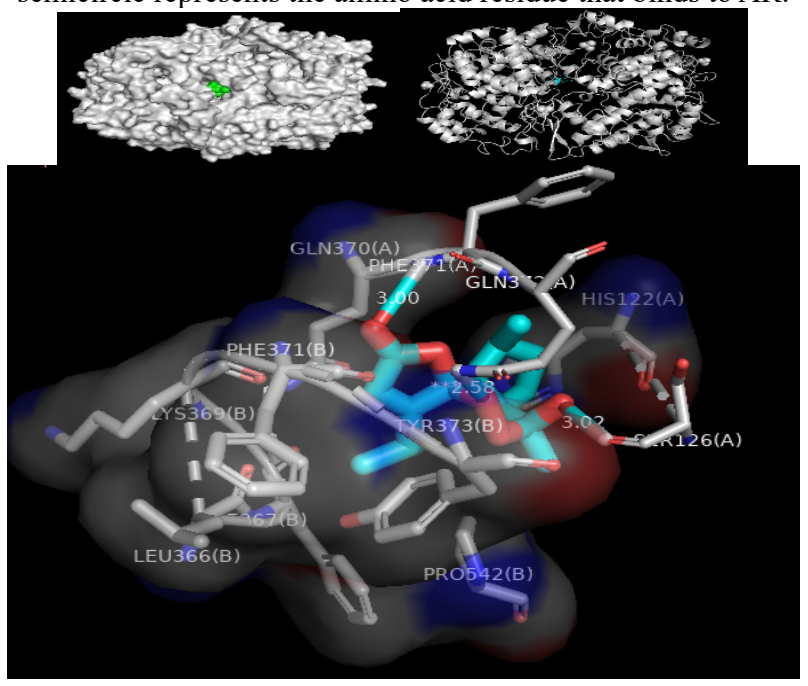

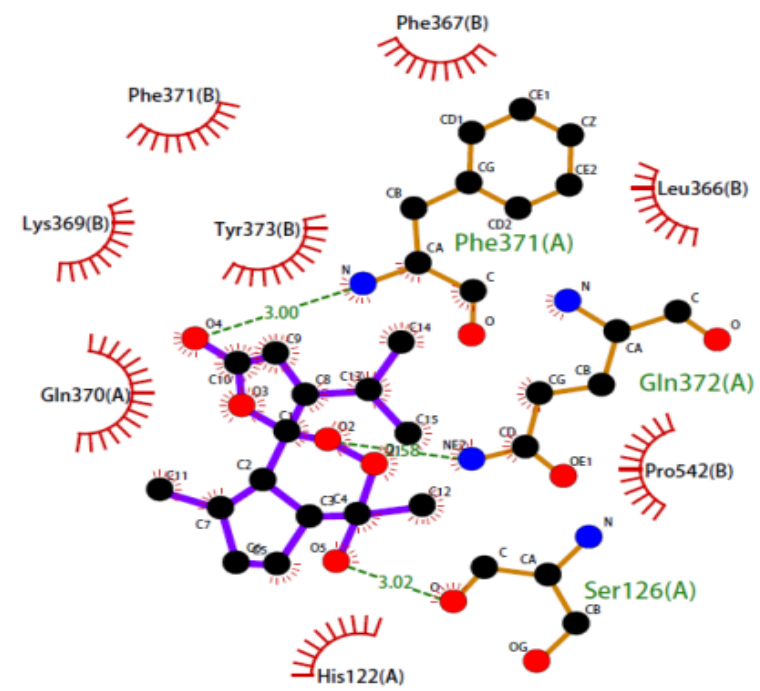

Fig.9-4 The Molecular models of DK binding to its predicted protein target PTGS2. Left 1, left 2:a global profile of massive possible docking, The off-white color indicates the receptor PTGS2, the green sphere and the blue line both indicate the ligand small molecule alpinolide peroxide.Left 3: the blue lines indicate the ligand small molecule alpinolide peroxide, the text represents the amino acid residue that binds to PTGS2.

Left4:the black nodes represent ligand small molecule alpinolide peroxide, the red semicircle represents the amino acid residue that binds to PTGS2.

\section{DISCUSSION}

Acne is a chronic inflammatory skin disease. Its pathogenesis is generally considered to be related to endocrine (especially androgen) factors, abnormal keratosis of sebaceous duct, microbial infection, diet or some drugs. There are a variety of pathogenic or non pathogenic microorganisms in acne lesions. The innate immune pattern recognition receptor of cellular immunity can recognize these microorganisms, especially Propionibacterium acnes, and then cause the immune response of the body. We used tcmsp, Batman, CTD, PDB and PubChem databases as well as a variety of Shengxin analysis methods to analyze the network pharmacology of cardamom. Finally, seven core active components including quercetin, giopinene, 7-hydroxyflavone, kaempferide peroxide, 1,7-diphenyl-5-hydroxy-6heptene-3-one, (2R) - 5,7-dihydroxy-2-phenylchroman4-one and dehydroisobutanol were found. Among them, quercetin, giopinin and 7-hydroxyflavone are all flavonoids, which have high medicinal value. (1) Anti free radical and anti-oxidation: studies have shown that the hydrogen atom of phenolic hydroxyl group can combine with peroxide free radical to form flavonoid free radical, which can further react with other free radicals to terminate the free radical chain reaction. Therefore, flavonoids can enhance the body's antioxidant effect and scavenging free radicals [13]. (2) Antibacterial effect: studies have shown that almost all flavonoids have different degrees of antibacterial activity [14]. (3) Regulating the role of animal hormones: flavonoids have the dual regulatory role of estrogen, can promote the growth of animals, affect the secretion and metabolism of sex hormones, and the level of hormones in the body. (4) 
Anti inflammatory and immunomodulatory effects: flavonoids have strong anti-inflammatory ability ${ }^{[15]}$. Xing nianzeng et al. Found that quercetin could inhibit the growth of transplanted tumor of prostate cancer cell line tram-c2 in mice. The mechanism may be related to inhibiting the expression of androgen receptor and promoting the apoptosis of tumor cells, indicating that quercetin can also regulate the secretion of androgen. Lim young hee et al. ${ }^{[16]}$ detected that kaempferol inhibited the growth of Propionibacterium acnes in a concentration dependent manner. In addition, indirect studies found that kaempferol and quercetin combined with clindamycin or erythromycin had synergistic inhibitory effect on Propionibacterium acnes in vitro, and flavonoids combined with antibiotics also had inhibitory effect on antibiotic resistant Propionibacterium acnes. Direct research found that flavonoids extracted from traditional Chinese medicine, including quercetin, had inhibitory effect on Propionibacterium acnes ${ }^{[17,18]}$. As a dihydroflavonoid, it can scavenge free radicals and inhibit Propionibacterium acnes. Habsah et al. Found that 1,7-diphenyl-5-hydroxy-6hepten-3-one also had DPPH radical scavenging activity when studying the methanol extract of Alpinia officinarum ${ }^{[19]}$. Dehydrodiisobutanol is a kind of benzofuran lignans, which has been reported to have antibacterial, antioxidant and anti-inflammatory effects ${ }^{[20]}$. Dehydrodisoeugenol can also significantly inhibit the phosphorylation dependent proteolysis of LPS stimulated inhibitor kappaB - $\alpha$ and the transcription activity of NF $-\kappa \mathrm{B}$ in cells ${ }^{[21]}$. These findings indicate that dehydrodiisobutanol can be used as an effective anti-inflammatory drug. Therefore, cardamom can play a role in the treatment of acne through multiple active ingredients, which reflects the characteristics of multi-component treatment of traditional Chinese medicine.

In PPI analysis and module analysis, we screened out four important targets of cardamom in the treatment of acne, AKT1 (degree = 13), F2 (degree = 11), AR and PTGS2 (AR and PTGS2 have the same connectivity, degree =9). AKT1 is a serine / threonine protein kinase, which has three closely related family members (AKT1, AKT2 and Akt3). It is involved in a variety of biological processes, such as cell proliferation, insulin signal transduction and angiogenesis. Akt activation depends on the activation of PI3K (phosphatidylinositol 3-kinase) pathway, which is related to the transcription of androgen receptor (AR) nuclear signal and cadherin signal transduction in keratinocytes. Studies have shown that androgen activates PI3K / Akt pathway through the interaction of $\mathrm{p} 85 \alpha, \mathrm{AR}$ and $\left.\mathrm{Src}{ }^{[22}\right]$. Clinical studies have shown that androgen levels and local androgen receptor (AR) increase leading to endocrine disorders is the main internal cause of acne, and there is a certain correlation between TCM syndromes. Therefore, androgen and its receptor changes are the endocrine basis of acne ${ }^{[23]}$. On the basis of disease differentiation, we emphasize the "combination of disease and syndrome", which can provide a new method for the clinical treatment of acne. Therefore, cardamom mainly through AKT1, F2, AR and PTGS2 and other multi-target collaborative play its role in the treatment of acne.

The results show that cardamom plays a role in the treatment of acne through multiple signaling pathways and regulation of a variety of biological functions, including
VEGF signaling pathway, PI3K Akt signaling pathway, inflammatory response, oxidative stress response and so on. Activated Akt can activate or inhibit a series of downstream substrates such as bad, caspase 9, NF - $\kappa \mathrm{B}$, gsk23 and so on through phosphorylation, thus regulating cell proliferation, differentiation, apoptosis and migration. Akt can also regulate nuclear factor kappa $\mathrm{B}(\mathrm{NF}-\kappa \mathrm{b})$ signal transduction by phosphorylating IKK $\alpha$ and tpl2. Zhang $\mathrm{Li}$ and other studies found that quercetin prevented the proliferation of prostate cancer PC3 cells by inhibiting the expression of VEGF and COX-2 ${ }^{[24]}$. As a natural flavonoid of 5,7-dihydroxyflavanone, vasopressin plays an anti-inflammatory role mainly by inhibiting transcription, MAPK signaling pathway and inflammatory cytokines (TNF - $\alpha$, IL-1 $\beta$, IL-6 and IL-10) ${ }^{[25]}$. The results showed that low concentration of giopine could inhibit the production of $\alpha$ - hemolysin and alleviate the cell damage caused by $\alpha$ - hemolysin. Dehydrodisoeugenol inhibited the activation of NF $-\kappa B$ and the expression of cyclooxygenase (COX) - 2 gene in LPS stimulated mouse macrophages ${ }^{[26]}$. Similarly, our study found that KEGG analysis of the main modules in PPI network was enriched into PI3K Akt and VEGF signaling pathways. Studies have found that PI3K Akt signaling pathway plays a key role in the development of acne. Drug treatment of acne vulgaris can improve microcirculation, reduce blood viscosity and promote skin repair. VEGF is a kind of vascular growth factor, which can promote wound healing and neovascularization. It can form epidermal layer earlier, which is conducive to the healing of skin lesions. Network pharmacology research also found that Angelica dahurica treatment of acne mainly involves PI3K Akt, NF - $\kappa$ B, MAPK, FGFR2 signaling pathway. Therefore, cardamom mainly through VEGF signaling pathway, PI3K Akt signaling pathway and other pathways involved in inflammatory response, endocrine regulation, oxidative stress response and other characteristics of multiple pathways to play its role in the treatment of acne.

The results of this study further explore the mechanism of cardamom in the treatment of acne vulgaris, and show that its treatment involves multiple active components, multiple targets and multiple signal pathways, and its mechanism is complex and diverse, not a single monomer, a target or a signal pathway, which is consistent with the multi-target and systematic nature of traditional Chinese medicine in the treatment of diseases. The results of molecular docking also confirmed the reliability of our network pharmacology research results. Of course, there are still some defects in this paper. In the follow-up work, we still need to further explore and verify the active ingredients, traditional Chinese medicine targets and pathways screened out in this paper, and then further explore more practical application value in favor of medical transformation through clinical practice.

Notes

The authors declare no competing financial interest. 


\section{ACKNOWLEDGMENTS}

This work was supported by grants from the Project No. 20171190 of Guangdong Administration of traditional Chinese Medicine

\section{REFERENCES}

1. Williams HC, Dellavalle RP, Garner S: Acne vulgaris. Lancet (London, England) 2012, 379(9813):361-372.

2. Hao Yan, Zhang Liang, Huang Kangbai, Zhang Xinpu: Discussion on the theoretical basis of pressing needle in the treatment of acne. Journal of Guangxi University of traditional Chinese medicine, 2018, 021 (4): p.5355

3. Zhang Ruojun: etiology and pathogenesis of acne. World's latest medical information abstracts 2016 (92): 30-32

4. Hopkins AL: Network pharmacology: the next paradigm in drug discovery. Nature chemical biology 2008, 4(11):682-690.

5. Chen Xiangjun, Liu Jing: network pharmacology mechanism of Angelica dahurica in treating acne. Journal of Guangzhou University of traditional Chinese medicine, 2019 (10):38-42

6. Liu Jun: network pharmacology of Salvia miltiorrhiza in the treatment of acne vulgaris. Guangdong Pharmaceutical University; 2015

7. Ru J, Li P, Wang J, Zhou W, Li B, Huang C, Li P, Guo Z, Tao W, Yang Y et al: TCMSP: a database of systems pharmacology for drug discovery from herbal medicines. Journal of cheminformatics 2014, 6:13

8. Liu Z, Guo F, Wang Y, Li C, Zhang X, Li H, Diao L, Gu J, Wang W, Li D et al: BATMAN-TCM: a Bioinformatics Analysis Tool for Molecular mechANism of Traditional Chinese Medicine. Scientific reports 2016, 6:211-215.

9. Davis AP, Grondin CJ, Johnson RJ, Sciaky D, McMorran R, Wiegers J, Wiegers TC, Mattingly CJ: The Comparative Toxicogenomics Database: update 2019. Nucleic acids research 2019, 47(D1):D948-d954.

10. Szklarczyk D, Franceschini A, Wyder S, Forslund K, Heller D, Huerta-Cepas J, Simonovic M, Roth A, Santos A, Tsafou KP et al: STRING v10: proteinprotein interaction networks, integrated over the tree of life. Nucleic acids research 2015, 43(Database issue):D447-452.

11. Tang Y, Li M, Wang J, Pan Y, Wu FX: CytoNCA: a cytoscape plugin for centrality analysis and evaluation of protein interaction networks. Bio Systems 2015, 127:67-72.

12. Kim S, Chen J, Cheng T, Gindulyte A, He J, He S, Li Q, Shoemaker BA, Thiessen PA, Yu B et al: PubChem 2019 update: improved access to chemical data. Nucleic acids research 2019, 47(D1):D1102-D1109.

13. Xiao Yongmei, Li Ming, Mao Pu, Yuan Jinwei: Research Progress on biological modification and activity of flavonoids. Journal of Henan University of
Technology (NATURAL SCIENCE EDITION) 2019, 040 (002): 123-131139

14. Adamczak A, Oarowski M, Karpi ń ski TM: Antibacterial Activity of Some Flavonoids and Organic Acids Widely Distributed in Plants. Journal of Clinical Medicine 2020, 9(1):34-38

15. Zheng Haiyan, Wang Xiaojie: Discussion on pharmacological action of flavonoids. Chinese Journal of biochemical medicine, 2017 (5):17-20

16. Lim YH, Kim IH, Seo JJ: In vitro activity of kaempferol isolated from the Impatiens balsamina alone and in combination with erythromycin or clindamycin against Propionibacterium acnes. Journal of microbiology (Seoul, Korea) 2007, 45(5):473-477.

17. Xia Mingjing, Wu Chenglong, et al. Inhibitory effect of 22 active components of Chinese herbal medicines on Propionibacterium acnes. Chin J dermatol 2001:59-72

18. Kim S, Oh S, Noh HB, Ji S, Lee SH, Koo JM, Choi CW, Jhun HP: In Vitro Antioxidant and AntiPropionibacterium acnes Activities of Cold Water, Hot Water, and Methanol Extracts, and Their Respective Ethyl Acetate Fractions, from Sanguisorba officinalis L. Roots. Molecules (Basel, Switzerland) 2018, 23(11).

19. Mohamad H, Abas F, Permana D, Lajis NH, Ali AM, Sukari MA, Hin TY, Kikuzaki H, Nakatani N: DPPH free radical scavenger components from the fruits of Alpinia rafflesiana Wall. ex. Bak. (Zingiberaceae). Zeitschrift fur Naturforschung C, Journal of biosciences 2004, 59(11-12):811-815.

20. Lv QQ, Yang XN, Yan DM, Liang WQ, Liu HN, Yang XW, Li F: Metabolic profiling of dehydrodiisoeugenol using xenobiotic metabolomics. Journal of pharmaceutical and biomedical analysis 2017, 145:725-733.

21. Murakami Y, Shoji M, Hirata A, Tanaka S, Yokoe I, Fujisawa S: Dehydrodiisoeugenol, an isoeugenol dimer, inhibits lipopolysaccharide-stimulated nuclear factor kappa $B$ activation and cyclooxygenase-2 expression in macrophages. Archives of biochemistry and biophysics 2005, 434(2):326-332.

22. Sun M, Yang L, Feldman RI, Sun XM, Bhalla KN, Jove R, Nicosia SV, Cheng JQ: Activation of phosphatidylinositol 3-kinase/Akt pathway by androgen through interaction of $\mathrm{p} 85 \alpha$, androgen receptor, and Src. The Journal of biological chemistry 2016, 291(43):22841.

23. Ju Q, Tao T, Hu T, Karadağ AS, Al-Khuzaei S, Chen $\mathrm{W}$ : Sex hormones and acne. Clinics in dermatology 2017, 35(2):130-137.

24. Zhang Li, Zhu Qingyi, Wei Yunfei, Yuan Lin, Ma long, Liu Li, Su Jian, Gu Xiaojian: effect of quercetin on the expression of VEGF and COX-2 in prostate cancer PC3 cells. New traditional Chinese medicine, 2012, 044 (1): 125-127 
25. Ye T, Zhang C, Wu G, Wan W, Liang J, Liu X, Liu D, Yang B: Pinocembrin attenuates autonomic dysfunction and atrial fibrillation susceptibility via inhibition of the NF- $\mathrm{kB} / \mathrm{TNF}-\alpha$ pathway in a rat model of myocardial infarction. International immunopharmacology 2019, 77:105-116.

26. Murakami Y, Shoji M, Hirata A, Tanaka S, Yokoe I, Fujisawa S: Dehydrodiisoeugenol, an isoeugenol dimer, inhibits lipopolysaccharide-stimulated nuclear factor kappa B activation and cyclooxygenase-2 expression in macrophages. Archives of Biochemistry and Biophysics 2005, 434(2):326-332. 